\title{
Benign metastasising leiomyoma of lung - two interesting cases
}

\author{
Authors: Akhilesh Sharma, Nazesh Javaid-Khan, Salma Haji-Hussein, Rizwan Kaiser and Urmi Gupta
}

\section{Introduction}

A leiomyoma, also known as a fibroid, is a benign smooth muscle tumour that very rarely becomes malignant $(0.1 \%)$. They can occur in any organ, but the most common forms occur in the uterus, small bowel and the oesophagus.

Benign metastasising leiomyoma $(\mathrm{BML})$ is a rare condition that occurs in all age groups, mostly between age 30 and 74 , and is particularly prevalent among women of late childbearing age. The most frequent sites of occurrence are the lungs and pelvis. The lesions are hormonally responsive. All BML patients usually have a history of uterine leiomyoma and/or myomectomy.

Steiner was the first to describe this disease in detail in 1939. He published a report of a patient who died from the effects of extensive pulmonary metastases of benign-appearing leiomyomas, which were histologically identical to the multiple leiomyomas in the uterus.

Some patients have symptoms such as a cough, dyspnoea or chest pain. BML is quite difficult to diagnose by simple medical imaging or physical examination and is often misdiagnosed as pneumonia, bronchitis or metastasising lung cancer. Most cases have been discovered by chest X-ray or computed tomography (CT) during routine examinations. Lung biopsy is the standard diagnostic procedure for BML.

We would like to report two interesting cases of BML. Our first case challenges the hypothesis of the haematogenous spread of leiomyoma after surgical intervention. In this case, BML in the lung developed simultaneously with uterine fibroid without having previous myomectomy/hysterectomy. The second case represents a rare case of recurrent leiomyoma.

\section{Case 1}

A 47-year-old nurse was being seen by her gynaecologist with a history of significant uterine leiomyomas and infertility. She presented acutely to the emergency department complaining of a few days' history of a productive cough, loss of appetite, fevers and night sweats. She is a non-smoker and does not drink alcohol, and was previously well and on no regular medication.

Initial investigations including a chest $\mathrm{X}$-ray were performed which demonstrated left-sided opacification suggestive of

Authors: Barking, Havering \& Redbridge University Hospitals NHS Trust, UK community-acquired pneumonia or possible tuberculosis (TB). Three sputum samples were sent off for acid-fast bacilli (AFB) smear analysis which came back negative. She was treated with antibiotics for pneumonia, which she reported improved her symptoms significantly. She had further investigations as an outpatient. Thorax CT demonstrated a $2 \mathrm{~cm}$ lobulated mass within the left main bronchus causing almost complete occlusion, associated pleural effusion and significant left-sided volume loss.

She had a fibre optic bronchoscopy which showed a near occluded left bronchus by an endobronchial tumour, with histology suggestive of being a leiomyoma. Histology from core biopsies undertaken at rigid bronchoscopy by thoracic surgeons confirmed diagnosis of leiomyoma, in keeping with a diagnosis of BML. She underwent a rigid bronchoscopy and endobronchial debulking therapy by thoracic surgeons to relieve the obstruction at the left bronchus. She subsequently underwent a myomectomy by gynaecologists for definitive treatment of her uterine leiomyoma. She was seen in the respiratory clinic for follow up where she reported no further respiratory symptoms. Follow-up chest X-ray showed chronic volume loss.

\section{Case 2}

A 52-year-old female and ex-smoker for over 10 years was referred under the 2-week wait following an incidental finding of a lung mass on routine chest $X$-ray following an acute presentation with chest pain. She had a background of ischaemic heart disease with previous myocardial infarction in 2014, type 2 diabetes, mild asthma and a hysterectomy in 2005 secondary to menorrhagia and fibroids.

Initial chest X-ray showed an ill-defined opacity in the right upper lobe which was initially presumed to be inflammatory but a neoplastic cause could not be excluded. During her assessment in clinic, she had no alarming symptoms and her clinical examination was unremarkable.

Chest CT showed a $3.7 \mathrm{~cm}$ spiculated area of consolidation in the right upper lobe and further smaller sub-centimetre nodules. She was discussed by the lung multidisciplinary team (MDT) and a CT-guided lung biopsy of the lung mass was performed. This found histology in keeping with pneumocyte hyperplasia. On positron emission tomography (PET) the lesion showed low-grade uptake with a standardised uptake value (SUV) max of 1.9. The other smaller sub-centimetre nodules in the right lung were non-fluorodeoxyglucose (FDG) avid. Given the ongoing concern for a potential malignant lesion, a repeat CT-guided lung biopsy 
was performed and showed histology of bronchoalveolar cell carcinoma.

She was then referred to the cardiothoracic surgeons and underwent a curative right upper lobectomy for a bronchoalveolar cell lung cancer (pathological staging T1 NO M0). In the pathology report it was noted that in the resected lobe in and around the tumour, there were tiny nodules of smooth muscle proliferation (smooth muscle actin (SMA), CD56 and CD10 positive), suggestive of a possible benign metastasising leiomyoma.

Following the curative resection, the patient was followed up with serial chest X-rays. At 2-year follow-up, chest X-ray showed possible new right apical changes. Chest CT and PET-CT were performed, and showed an increase in the number and size of the multiple pulmonary nodules ranging from $7 \mathrm{~mm}$ to $12 \mathrm{~mm}$. They remained non-avid on PET. The imaging findings were concerning for malignancy, either for recurrence or progression of the residual disease. Following discussion at the MDT she was referred to the thoracic surgeons and underwent a left videoassisted thoracic surgery (VATS) wedge resection of the largest nodule in the left lower lobe. The histology was of benign smooth muscle proliferation (SMA, CD10 and CD56 positive) in keeping with a possible diagnosis of BML. A referral was made to the gynaecologists to ascertain whether any hormonal therapy was indicated and/or surveillance screening.

\section{Discussion}

Pulmonary smooth muscle proliferation can either be primary, including hamartomas, lymphangioleiomyomatosis, leiomyoma and leiomyosarcoma, or metastatic, including metastatic leiomyosarcoma and BML. The low mitotic index $(<5$ mitoses per 10 high power fields), lack of nuclear pleomorphism, lack of local invasion and distinctive karyotypic profile helps differentiate BML from other possible diagnoses.

The majority of women with BML report having had a hysterectomy or myomectomy in the past due to leiomyomas. However, the changes in lungs may also occur before uterine surgery. The condition is usually characterised by a benign course. Cough, dyspnoea and chest pain are rarely present. The most common presentation is solitary or multiple pulmonary nodules. The average size of the pulmonary nodules ranges from 0.5 to $10 \mathrm{~cm}$. Most frequently, in $70 \%$ of the patients, the lesions in the lungs occur bilaterally and multiple unilateral lesions are seen in $17 \%$ of cases.

The hypotheses explaining the histogenesis and metaplastic mechanisms are subject to some controversy. In the past, they were described as multiple fibroleiomyomatous hamartomas, assuming they developed de novo in the lungs. Currently, the majority of authors regard them as haematogenous metastases originating from benign leiomyomas with likely increased mitotic activity. Our first case report challenges this hypothesis. In the first case, the patient had simultaneous pulmonary fibroid along with leiomyoma of the uterus.

According to some, primary uterine tumours in these cases should be classified as low-grade leiomyosarcoma, due to their ability to metastasise. On the other hand, their benign microscopic appearance and clinical course contradict such a hypothesis. It has also been suggested that smooth muscle proliferation occurring in various organs might result from the improper hormonal status.

BML should not be mistaken for lymphangioleiomyomatosis which is a disease caused by the proliferation of smooth muscle cells in the lymphatic vessels of the lungs and lymph nodes.

Due to the rarity of the disease, currently, there are no treatment guidelines for BML. Multiple treatment options have been reported in the literature, including close observation, surgical resection or antioestrogen therapy (eg selective oestrogen receptor modulator, progesterone, aromatase inhibitors, oophorectomy and gonadotropin-releasing hormone analogues). The preferred treatment is surgical resection if possible, with hormonal therapy as an alternate. Our first case had curative treatment for an endobronchial tumour and myomectomy. The second case had curative upper lobe resection and was referred to gynaecology to be considered for hormonal treatment.

BML tends to typically have an indolent course and a favourable outcome, although pulmonary lesions may continue to progress, resulting in pulmonary insufficiency and even death.

\section{Conclusion}

> Although rare, uterine leiomyomas can metastasise to the lung.

> Despite BML being a rare condition, it should be considered as the differential diagnosis in women of reproductive age with a history of uterine leiomyoma presenting with pulmonary nodules, either solitary or multiple.

> The diagnosis of BML should also be considered in cases of pulmonary nodules in young women with no previous history of uterine surgery.

> BML can recur after curative treatment and it should be considered as differential diagnosis in case of recurrence of an endobronchial tumour.

\section{Conflict of interest statement}

None declared. 